\title{
ANDES

www.scielo.cl

\section{Dolor en pacientes pediátricos hospitalizados en una institución de salud de tercer nivel}

\author{
Pain in hospitalized pediatric patients in a third-level healthcare institution
}

\author{
Iván Darío Zúñiga-Espitia ${ }^{a}$
}

aPrograma de Especialización en Epidemiología, Facultad de Ciencias para la Salud, Universidad de Caldas. Manizales, Colombia

Recibido: 31 de marzo de 2020; Aceptado: 31 de mayo de 2021

¿Qué se sabe del tema que trata este estudio?

Al comprender el dolor como un fenómeno comunicacional y subjetivo, puede ser habitual que no se indague, no se trate e incluso se ignore, pero la incapacidad del niño para expresarlo verbalmente, no debe negarle la posibilidad de recibir manejo.

\begin{abstract}
¿Qué aporta este estudio a lo ya conocido?
En comparación con otros grupos de edad, los estudios publicados sobre dolor en pacientes pediátricos son menos frecuentes, este estudio aporta información sobre la caracterización y el manejo del dolor en niños en un entorno hospitalario de una ciudad Latinoamericana.
\end{abstract}

\section{Resumen}

En el ámbito hospitalario, los pacientes pediátricos pueden presentar cuadros clínicos dolorosos o ser sometidos a procedimientos que generan dolor, por lo tanto, reconocer su existencia y realizar un abordaje clínico de este debe ser una prioridad en los equipos de salud. Objetivo: Caracterizar el dolor y su abordaje clínico-terapéutico en pacientes hospitalizados en la sala general de una institución pediátrica de tercer nivel de atención. Pacientes y Método: Estudio de tipo observacional, transversal, realizado entre agosto y octubre de 2019. Se incluyeron 187 pacientes. Variables de estudio: sexo, motivo de hospitalización, especialidad tratante, dolor crónico, actual y en las 24 horas previas, intensidad, causa máxima de dolor, prescripción analgésica. Se utilizó en niños entre los dos meses y tres años de edad la escala FLACC (Face, Legs, Activity, Cry, Consolability), en niños entre cuatro y ocho años la escala de caras revisada y a partir de los nueve años la escala visual analógica de autoevaluación del dolor. Posterior al análisis de los datos con estadísticos descriptivos, se calcularon las diferencias entre grupos con prueba $\chi^{2}$ para variables cualitativas, pruebas no paramétricas para comparación de grupos con variable ordinal. Resultados: el 10,7\% de los pacientes presentaban dolor crónico, sin diferencias según el sexo $(\mathrm{p}=0,713)$. La prevalencia de dolor al momento de la evaluación fue del $38,5 \%$, sin diferencias según el sexo $(p=0,419)$. Pacientes que ingresaron por cirugía y trauma presentaron mayor prevalencia de dolor $(\mathrm{p}=0,034)$ y el $53,5 \%$ de los menores tenían prescripción analgésica. Conclusión: La prevalencia del dolor en el paciente pediátrico hospitalizado es alta. Adicionalmente se reporta alto uso de analgesia no opioide y baja prescripción de analgésicos opioides en el entorno pediátrico para el manejo habitual del dolor de intensidad moderada y severa.
Palabras clave:

Dolor;

Niño;

Manejo del Dolor;

Niño Hospitalizado;

Analgesia 


\begin{abstract}
In the hospital setting, pediatric patients can present painful conditions or undergo procedures that generate pain, therefore, recognizing their existence and carrying out a clinical approach to it should be a priority in health care teams. Objective: To characterize the pain and its clinical-therapeutic approach in hospitalized patients in the general ward of a tertiary pediatric healthcare institution. Patients and Method: An observational, cross-sectional, and descriptive study conducted between August and October 2019. 187 patients were included. Study variables were sex, reason for hospitalization, treating specialty, chronic, current, and in the last 24 hours pain, intensity, maximum cause of pain, and analgesic prescription. The FLACC (Face, Legs, Activity, Cry, Consolability) scale was used in children aged between two months and three years, the revised Faces Pain Scale in children between four and eight years of age, and the self-reported Visual Analogue Scale was used in children from nine years of age onwards. After data analysis with descriptive statistics, differences between groups were calculated with the Chi-square test for qualitative variables, and non-parametric tests for comparison of groups with ordinal variables. Results: $10.7 \%$ of the patients had chronic pain, without differences according to $\operatorname{sex}(\mathrm{p}=0.713)$. The prevalence of pain at the time of the evaluation was $38.5 \%$, without differences according to sex $(p=0.419)$. Patients admitted for surgery and trauma had a higher prevalence of pain $(p=0.034)$ and $53.5 \%$ of the children had an analgesic prescription. Conclusion: the prevalence of pain in the hospitalized pediatric patients is high. Additionally, high use of non-opioid analgesia and a low prescription of opioid analgesics in the pediatric sphere is reported for the usual management of moderate and severe intensity pain.
\end{abstract}

Keywords:

Pain;

Child;

Pain Management;

Hospitalized Child;

Analgesia

\section{Introducción}

En el proceso neurofisiológico del dolor, la activación de los nociceptores secundario a la presencia de un estímulo nocivo genera potenciales de acción a nivel de estos receptores sensoriales periféricos que se transmite a través de fibras aferentes hacia el sistema nervioso central, para convertirse en una percepción consciente del dolor ${ }^{1}$. Estudios sobre el desarrollo de la neurobiología del dolor ${ }^{2}$ han demostrado que el ser humano tiene una maduración considerable de la transmisión del dolor aferente periférico, espinal y supraespinal a partir de la semana 26 de gestación ${ }^{3,4}$ capaz de ser codificado y que responde a daño tisular con factores autonómicos, hormonales y signos de estrés metabólico ${ }^{5}$. Por lo tanto, el dolor se reconoce como una experiencia universal y un problema global ${ }^{6}$, principalmente en poblaciones vulnerables como la población pediátrica; en la cual la incapacidad del niño para expresar verbalmente el dolor, no debe negar la existencia y necesidad de alivio ${ }^{7}$. Desde la Declaración de Montreal, publicada por la International Association for the Study of Pain (IASP) y la Organización Mundial de la Salud (OMS) en el 2010, se establece que el acceso al tratamiento del dolor es un derecho humano fundamental ${ }^{8}$.

El dolor se debe considerar como el quinto signo vital $^{9}$ y evaluarse en todos los pacientes, con el propósito de promover un manejo adecuado. La mayoría de las etiologías de dolor se pueden prevenir, tratar o, al menos, reducir utilizando las herramientas terapéuti- cas farmacológicas y no farmacológicas disponibles ${ }^{10}$. Existen medios y conocimientos suficientes para aliviar el dolor; por lo tanto, reconocer su existencia y realizar un abordaje clínico debe ser prioridad de los equipos de salud.

En el ámbito hospitalario, los pacientes pueden presentar padecimientos dolorosos o ser sometidos a procedimientos que generan dolor. Por lo que es común, que los clínicos se enfrenten de forma cotidiana a pacientes que requieren su manejo; en especial en pediatría, donde el dolor no tratado puede tener efectos fisiológicos adversos ${ }^{11}$, retrasar la recuperación e impactar negativamente tanto a corto como a largo plazo en la salud física y emocional de los pacientes, hasta el punto de modificar la forma en la que el sistema nervioso del niño percibirá el dolor en el futuro ${ }^{12,13}$. A su vez, diferentes consensos han llegado a la conclusión que aspectos como la variabilidad clínica y cognitiva deben entrar a ser considerados e instaurar estos tratamientos con la mínima demora posible, adaptándolos a las características individuales de cada paciente ${ }^{14,15}$.

En Latinoamérica, existen pocos estudios relacionados con dolor en pacientes pediátricos en entornos hospitalarios. Uno de ellos fue realizado en el Hospital Pediátrico del Centro Hospitalario Pereira Rossell (HP-CHPR) y otras instituciones públicas y privadas de Montevideo (Uruguay), publicado en el año 2016, reporta una prevalencia del dolor en niños hospitalizados del 15,5\% al momento de la evaluación y del 35\% en las $24 \mathrm{~h}$ previas, otro estudio en el mismo hospital publicado en el 2018, reporta una prevalencia similar ${ }^{17}$. 
El presente estudio tiene como objetivo caracterizar la etiología, intensidad y abordaje clínico-terapéutico del dolor en pacientes hospitalizados de una institución pediátrica de tercer nivel de atención.

\section{Pacientes y Método}

\section{Diseño}

Se llevó a cabo una investigación de tipo observacional, prospectiva, transversal y descriptiva con pacientes hospitalizados en sala general de una institución pediátrica de tercer nivel de atención de la ciudad de Manizales, Colombia. La muestra se calculó a partir de una prevalencia del $50 \%$, por no conocerse la prevalencia real del dolor, y según el total de egresos hospitalarios promedios en un mes calendario. Se incluyeron 187 pacientes con estancia no inferior a $24 \mathrm{~h}$ desde el ingreso, se excluyeron los menores de dos meses de edad y aquel paciente y/o cuidador ausente después de tres intentos para efectuar la evaluación.

\section{Instrumentos utilizados}

Se realizó la valoración del paciente y la recolección de información mediante un instrumento tipo encuesta y revisión de historias clínicas entre los meses de agosto y octubre del 2019. A los pacientes y/o cuidadores se les preguntó si el paciente había experimentado algún dolor desde el ingreso al hospital, se consideró paciente con dolor cuando el niño, adolescente y/o cuidador refirieron que el menor tenía dolor en el momento de la evaluación o había tenido en las $24 \mathrm{~h}$ previas. Se interrogó si estaban informados sobre tratamiento para el dolor y si habían solicitado asistencia médica para aliviar el dolor en las últimas $24 \mathrm{~h}$.

La evaluación del dolor fue obtenida del propio paciente a través de escalas validadas recomendadas por la OMS. Se utilizó en niños entre los dos meses y tres años de edad la escala FLACC (Face, Legs, Activity, Cry, Consolability $)^{19}$, en niños entre cuatro y ocho años la escala de caras revisada ${ }^{20,21}$ y a partir de los nueve años la escala visual analógica de autoevaluación del dolor ${ }^{22}$. Se empleó una ficha diseñada para el estudio, con la cual se hizo una prueba piloto en las instalaciones del centro hospitalario durante dos días no consecutivos, semanas previas al estudio; ajustado a los criterios de selección definidos, esto permitió saber el tiempo requerido por cada paciente. Además se hizo necesario efectuar modificaciones en la terminología empleada en la ficha para facilitar la comprensión por pacientes y/o acudientes. La encuesta incluía las siguientes variables: sexo, edad, motivo de hospitalización, tiempo de estancia hospitalaria, especialidad tratante, dolor previo y actual, intensidad del dolor, causa máxima de dolor, registro de presencia de dolor y calidad del re- gistro, prescripción analgésica actual, solicitud previa de analgesia. La información obtenida del estudio está bajo confidencialidad del equipo investigador, garantizando el manejo adecuado y discrecional del dato.

\section{Análisis estadístico}

Se consolidó la información en una matriz del programa IBM SPSS Statistics $22^{\circledR}$. Se efectuó un análisis inicial de las características sociodemográficas con estadísticos descriptivos para resumir los datos, con medidas de tendencia central y dispersión; las frecuencias y porcentajes se usaron para las variables categóricas. Se calculó la diferencia de medias de edad entre ambos sexos con prueba t-student; como también, se usó la prueba $\chi^{2}$ para establecer diferencias entre grupos de interés con variables cualitativas. Se aplicó la prueba no paramétrica de U de Mann-Whitney para comparación de dos grupos con variable ordinal y la prueba de Kruskal-Wallis para comparar más de dos grupos con variables ordinales. Además se comparó la prevalencia del dolor según la especialidad tratante y según motivo de ingreso a hospitalización con prueba $\chi^{2}$ para comparar más de dos grupos y posteriormente se realizó recodificación en distintas variables según la causa de la significancia. Se consideró significativo si p $<0,05$ para todas las pruebas estadísticas.

\section{Aspectos éticos}

El proyecto fue aprobado por el comité de ética de la Facultad de Ciencias para la Salud de la Universidad de Caldas Acta No 008 de 2019 y el comité de investigación de la institución pediátrica donde se llevó a cabo. La participación fue voluntaria, previa obtención de consentimiento informado de los padres o acudientes del paciente; la negación a participar no interfirió de ninguna manera con la atención del paciente por parte del equipo asistencial.

\section{Resultados}

Se incluyeron 187 pacientes, 77 de sexo femenino y 110 de sexo masculino; la mediana de edad fue de 96 meses (RIQ 34-165 meses), sin diferencias significativas según el sexo $(\mathrm{p}=0,169)$ y con una media de estancia hospitalaria de 2,89 $(2,20 ; 3,57)$ días. El principal motivo de ingreso hospitalario fue la patología no infecciosa en el 30,5\% de los pacientes y sin diferencias según sexo $(\mathrm{p}=0,128)$ como se describe en la tabla 1 .

En cuanto al dolor, el 10,7\% de los pacientes presentaban dolor crónico, definido como dolor de tres meses o más de duración, previo al ingreso hospitalario, sin encontrarse diferencias estadísticamente significativas entre ambos sexos ( $p=0,713)$, y fue el dolor abdominal la causa más frecuente de dolor crónico, en 
un $40 \%$ de los pacientes. Al momento de la evaluación, el 38,5\% de los pacientes presentaban dolor, sin diferencias según sexo $(\mathrm{p}=0,419)$ y el motivo de ingreso hospitalario no generó diferencias significativas en la intensidad del dolor actual ( $\mathrm{p}=0,093)$ (tabla 2).

Así mismo, al momento de la evaluación, la prevalencia de dolor fue mayor en pacientes con antecedente de dolor crónico $(\mathrm{p}=0,002)$, con una incidencia del $70 \%$ de dolor actual entre pacientes con dolor crónico en comparación con el 34,7\% de pacientes sin antecedente de dolor crónico que estaban álgicos al momento de la evaluación. Se utilizó la escala FLACC en 16 pacientes, escala de dolor con caras - Revisada (FPS-R) en 12 pacientes y escala visual analógica en 44 pacientes, sin encontrarse diferencias estadísticamente significativas en la intensidad de dolor según el grupo de edad y/o escala utilizada $(\mathrm{p}=0,000)$.

Los pacientes manejados por ortopedia presentaron una mayor prevalencia de dolor $(\mathrm{p}=0,005)$, pero sin diferencias significativas en la intensidad $(\mathrm{p}=0,11)$ comparado con las otras especialidades; como también, los pacientes que ingresaron por cirugía y trauma presentaron mayor prevalencia de dolor al momento de la valoración $(\mathrm{p}=0,034)$ como se detalla en la tabla 3 .

Se documentó que el 59,9\% de los pacientes habían presentado algún dolor en las $24 \mathrm{~h}$ previas a la evaluación, sin diferencias según el sexo $(\mathrm{p}=0,972)$ ni motivo de ingreso hospitalario $(\mathrm{p}=0,168)$. De los pacientes con dolor previo, el 54,5\% fue de leve intensidad y el $12,5 \%$ correspondía a dolor severo. Además el 13,9\% de los 187 pacientes y/o acudientes habían solicitado al personal asistencial alguna medida adicional para ayudar a aliviar el dolor en las 24 h previas a la evaluación; al mismo tiempo, el 26,7\% refirieron no haber presentado dolor en ningún momento durante la estancia hospitalaria, en contraste con el 73,3\% de pacientes que habían experimentado dolor en algún momento durante la hospitalización, cuya causa de máximo dolor fue la punción con agujas como se muestra en la tabla 4.

Por otro lado, el 78,1\% de los pacientes y/o acudientes manifestaron que algún miembro del personal asistencial había indagado sobre dolor en el paciente en las $24 \mathrm{~h}$ previas a la evaluación y el $71,1 \%$ de los pacientes tenían registro en la historia clínica sobre existencia o no de dolor en este mismo periodo de tiempo (tabla 4).

Con respecto a la prescripción analgésica, el 53,5\% tenían prescripción analgésica de algún tipo como se resumen en la tabla 5 . De estos, el $98 \%$ eran tratados con analgésicos no opioides y el $2 \%$ restante con opioides, uno con opioide débil (tramadol) y otro con opioide potente (morfina), ambos en combinación con un medicamento no opioide, acetaminofén.
Tabla 1. Características sociodemográficas y motivo de ingreso de los pacientes

\begin{tabular}{lccc}
\hline Variables & $\begin{array}{c}\text { Frecuencia } \\
n=187\end{array}$ & $\begin{array}{c}\text { Porcentaje } \\
\%\end{array}$ & $p$ \\
\hline Sexo & & & \\
$\quad$ Femenino & 77 & 41,2 & \\
Masculino & 110 & 58,8 & \\
Edad & & & $0,169 *$ \\
3-11 meses & 13 & 7,0 & \\
12-23 meses & 21 & 11,2 & \\
24-71 meses & 44 & 23,5 & \\
72-144 meses & 41 & 21,9 & \\
144-215 meses & 68 & 36,4 & \\
Motivo de ingreso & & & $0,128^{* *}$ \\
Patología no infecciosa & 57 & 30,5 & \\
Patología infecciosa & 45 & 24,1 & \\
Cirugía o postoperatorio & 38 & 20,3 & \\
Para procedimientos o estudios & 24 & 12,8 & \\
Trauma & 18 & 9,6 & \\
Quemadura & 5 & 2,7 & \\
\hline
\end{tabular}

* La diferencia por sexo fue estimada con t-student para muestras independientes. **Para variables cualitativas la diferencia entre sexo fue estimada con la prueba $\chi^{2}$ de homogeneidad. Fuente: Elaboración propia.

Tabla 2. Prevalencia, localización e intensidad del dolor al momento de la evaluación

\begin{tabular}{|c|c|c|c|}
\hline Variables & $\begin{array}{c}\text { Frecuencia } \\
\mathrm{n}=187\end{array}$ & $\begin{array}{c}\text { Porcentaje } \\
\%\end{array}$ & $p$ \\
\hline $\begin{array}{l}\text { Dolor actual } \\
\text { Sí } \\
\text { No }\end{array}$ & $\begin{array}{r}72 \\
115\end{array}$ & $\begin{array}{l}38,5 \\
61,5\end{array}$ & \\
\hline $\begin{array}{l}\text { Dolor actual según sexo } \\
\text { Femenino } \\
\text { Si dolor actual } \\
\text { No dolor actual }\end{array}$ & $\begin{array}{l}27 \\
50\end{array}$ & $\begin{array}{l}35,1 \\
64,9\end{array}$ & $0,419 *$ \\
\hline $\begin{array}{l}\text { Masculino } \\
\text { Si dolor actual } \\
\text { No dolor actual }\end{array}$ & $\begin{array}{l}45 \\
65\end{array}$ & $\begin{array}{l}40,9 \\
59,1\end{array}$ & \\
\hline $\begin{array}{l}\text { Intensidad del dolor } \\
\text { Leve } \\
\text { Moderado } \\
\text { Severo }\end{array}$ & $\begin{array}{r}44 \\
21 \\
7\end{array}$ & $\begin{array}{r}61,1 \\
29,2 \\
9,7\end{array}$ & $0,244 * *$ \\
\hline $\begin{array}{l}\text { Localización de dolor actual } \\
\text { Abdomen } \\
\text { Cabeza } \\
\text { Miembro inferior } \\
\text { Orofaríngeo } \\
\text { Genitales } \\
\text { Miembros superior } \\
\text { Tórax } \\
\text { Cintura escapular } \\
\text { Oído } \\
\text { Cadera } \\
\text { Lumbar }\end{array}$ & $\begin{array}{r}24 \\
10 \\
10 \\
6 \\
5 \\
5 \\
4 \\
3 \\
2 \\
2 \\
1\end{array}$ & $\begin{array}{r}33,3 \\
13,9 \\
13,9 \\
8,3 \\
6,9 \\
6,9 \\
5,6 \\
4,2 \\
2,8 \\
2,8 \\
1,4\end{array}$ & $0,365^{*}$ \\
\hline
\end{tabular}


Tabla 3. Prevalencia del dolor según especialidad médica tratante y según motivo de ingreso

\begin{tabular}{|c|c|c|c|c|c|}
\hline \multirow[t]{3}{*}{ Variables } & \multicolumn{4}{|c|}{ Dolor actual } & \multirow[t]{3}{*}{$\mathrm{p}$} \\
\hline & \multicolumn{2}{|c|}{ Sí } & \multicolumn{2}{|c|}{ No } & \\
\hline & $n$ & $\%$ & $n$ & $\%$ & \\
\hline Especialidad médica tratante & & & & & $0,005^{*}$ \\
\hline Pediatría & 19 & 25,7 & 55 & 74,3 & \\
\hline Cirugía pediátrica & 20 & 42,6 & 27 & 57,4 & \\
\hline Pediatría y otra especialidad & 11 & 40,7 & 16 & 59,3 & \\
\hline Ortopedia & 14 & 73,7 & 5 & 26,3 & \\
\hline Cirugía pediátrica y otra especialidad & 3 & 33,3 & 6 & 66,7 & \\
\hline Pediatría y cirugía pediátrica & 2 & 33,3 & 4 & 66,4 & \\
\hline Ortopedia y otra especialidad & 2 & 100 & 0 & 0 & \\
\hline Pediatría y ortopedia & 1 & 50 & 1 & 50 & \\
\hline Cirugía plástica & 0 & 0 & 1 & 100 & \\
\hline Motivo de ingreso & & & & & $0,034^{*}$ \\
\hline Patología no infecciosa & 18 & 31,6 & 39 & 68,4 & \\
\hline Patología infecciosa & 14 & 31,1 & 31 & 68,9 & \\
\hline Cirugía o postoperatorio & 21 & 55,3 & 17 & 44,7 & \\
\hline Para procedimientos o estudios & 9 & 37,5 & 15 & 62,5 & \\
\hline Trauma & 10 & 55,6 & 8 & 44,4 & \\
\hline Quemadura & 0 & 0,0 & 5 & 100,0 & \\
\hline
\end{tabular}

* La diferencia en la prevalencia de dolor según especialidad médica o según motivo de ingreso fue estimada con la prueba $\chi^{2}$ de homogeneidad. Fuente: Elaboración propia.

\begin{tabular}{|c|c|c|}
\hline Variables & $\begin{array}{c}\text { Frecuencia } \\
n\end{array}$ & $\begin{array}{c}\text { Porcentaje } \\
\%\end{array}$ \\
\hline \multicolumn{3}{|l|}{ Causa de máximo dolor } \\
\hline Punción de agujas & 46 & 33,6 \\
\hline Postoperatorio - herida quirúrgica & 44 & 32,1 \\
\hline Movilización - terapia física & 25 & 18,2 \\
\hline Comer-odinofagia & 9 & 6,6 \\
\hline Procedimientos diagnósticos & 6 & 4,4 \\
\hline Deposición y/o micción & 6 & 4,4 \\
\hline Toser & 1 & 0,7 \\
\hline \multicolumn{3}{|l|}{ Registro de dolor } \\
\hline Sí & 133 & 71,1 \\
\hline No & 54 & 28,9 \\
\hline \multicolumn{3}{|l|}{ Personal de salud que registra el dolor } \\
\hline Enfermería & 26 & 19,5 \\
\hline Medico & 26 & 19,5 \\
\hline Médico y enfermería & 81 & 60,9 \\
\hline \multicolumn{3}{|l|}{ Calidad del registro } \\
\hline Completo & 99 & 74,4 \\
\hline Incompleto & 34 & 25,6 \\
\hline
\end{tabular}

Fuente: Elaboración propia.
El medicamento más empleado fue el acetaminofén, indicado en el $78 \%$ de los pacientes que recibían analgesia y representó el 54,9\% de las prescripciones y en menor frecuencia los opioides, en el 1,4\% de las prescripciones e indicado solo en el $2 \%$ de los pacientes que recibieron analgesia (tabla 5).

Entre los fármacos antiinflamatorios no esteroideos (AINEs), el más utilizado fue el ibuprofeno en el $50 \%$ de los menores que recibieron AINEs, diclofenaco en el $25 \%$, naproxeno y nimesulida en el $12,5 \%$ cada uno. Entre los pacientes que estaban con monoterapia analgésica, el 65,6\% recibieron acetaminofén y el $34,4 \%$ metamizol. De los que tenían dos medicamentos prescritos, el 52,8\% recibieron acetaminofén-metamizol y el 100\% de pacientes que recibieron tres medicamentos analgésicos tenían acetaminofén, metamizol y bupivacaína, cuatro pacientes requirieron analgesia de rescate en las $24 \mathrm{~h}$ previas a la evaluación, de los cuales el 50\% recibió analgesia con hidromorfona.

En relación con la vía de administración, en el 81\% de los pacientes que tenían prescripciones analgésicas se utilizó la vía oral, seguida de la vía parenteral periférica en el $52 \%$, epidural $6 \%$ y solo en el $1 \%$ de los pacientes se indicó la vía intramuscular para la administración de analgesia. Se debe agregar que del total de prescripciones de analgesia farmacológica, el 9,15\% fue prescrita para administración sin horario y/o a demanda del paciente. 


\section{Discusión}

Dada la importancia del reconocimiento del dolor en pediatría, a menudo subdiagnósticado en el paciente hospitalizado. Se realiza este estudio, que muestra una prevalencia de dolor al momento de la evaluación del $38,5 \%$, sin diferencias significativas entre ambos sexos $(\mathrm{p}=0,419)$; al comparar esta prevalencia con estudios en otros países latinoamericanos, es similar a la reportada por López-Guzmán y cols. en un estudio realizado en México en el 2013 que reportó una prevalencia del $35,3 \%{ }^{23}$. La prevalencia del presente proyecto fue mayor a la reportada por Zunino y cols. de Uruguay en el 2016 y 2018 que fue de 15,5\% y 15,8\% respectivamen$\mathrm{te}^{16,17}$, lo cual podría estar relacionada con la diferencia en el diseño de la investigación.

Es más frecuente el dolor actual en aquellos pacientes que ingresaron por trauma y cirugía $(\mathrm{p}=0,03)$, con una prevalencia del $55,6 \%$ y $55,3 \%$ respectivamente, situación que podría estar relacionada con el tipo de lesión tisular que genera el trauma o la patología quirúrgica asociado a estímulos nocivos, respuesta inflamatoria y mayor nocicepción, respecto a otros motivos de ingreso hospitalario ${ }^{24,25}$.

Por otro lado, el estudio reporta una prevalencia de dolor crónico del 10,7\%, sin diferencias estadísticamente significativas entre sexos $(p=0,713)$, hay que tener en cuenta que este tipo de dolor no es un problema exclusivo de la población adulta, en los pacientes pediátricos ha aumentado, convirtiéndose en una condición de salud común en población joven, con una mediana de prevalencia de acuerdo a la localización del dolor del 11\% al 38\% según lo descrito por Liossi C. y $\mathrm{cols}^{26}$.

Es importante resaltar que el 59,9\% de los pacientes había presentado algún tipo de dolor en las 24 h previas a la evaluación, en contraste con lo publicado por Friedrichsdorf y cols. en una investigación realizada en Estados Unidos en el 2015, en el cual el 76\% de los pacientes había experimentado dolor durante las $24 \mathrm{~h}$ previas $^{27}$. En esa mismo publicación, el peor dolor reportado fue causado por la punción con agujas en el $40 \%$ de los pacientes ${ }^{27}$, similar a lo encontrado en el actual estudio, en el que el motivo más frecuente de máximo dolor fue también la punción con agujas para realización de paraclínicos y/o administración de medicamentos en el 33,6\% de los pacientes. Además de resaltar que en ambas publicaciones más del 50\% de los pacientes tuvieron dolor previo a la evaluación.

Al mismo tiempo se estableció que el 13,9\% de los pacientes habían solicitado al personal asistencial, alguna medida adicional para ayudar a aliviar el dolor en las $24 \mathrm{~h}$ previas a la evaluación y que el $26,7 \%$ de los pacientes no habían presentado ningún dolor durante la estancia hospitalaria. Lo anterior podría estar relacio-
Tabla 5. Percepción y tipo de prescripción analgésica

\begin{tabular}{lcc}
\hline Variables & $\begin{array}{c}\text { Frecuenciaa } \\
n\end{array}$ & $\begin{array}{c}\text { Porcentaje } \\
\%\end{array}$ \\
\hline Percepción sobre prescripción analgésica & & \\
Creen tener prescripción analgésica & 101 & 54,0 \\
Desconocen si tienen prescripción analgésica & 44 & 23,5 \\
Aseguran no tener prescripción analgésica & 42 & 22,5 \\
Prescripción analgésica & & \\
Sí & 100 & 53,5 \\
No & 87 & 46,5 \\
Tipo de prescripción & & \\
Farmacológica & 94 & 94 \\
Farmacológica y no farmacológica & 6 & 6 \\
Número de fármacos analgésicos & & \\
Un medicamento & 61 & 61 \\
Dos medicamentos & 36 & 36 \\
Tres medicamentos & 3 & 3 \\
Número de prescripciones por tipo de medicamento & \\
Acetaminofén & 78 & 54,9 \\
Dipirona & 48 & 33,8 \\
AlNES & 8 & 5,6 \\
Bupivacaína & 6 & 4,2 \\
Opioides & 2 & 1,4 \\
\hline
\end{tabular}

Fuente: Elaboración propia.

nado con aquellas situaciones médicas que requieren manejo intrahospitalario sin ser generadoras de dolor, sumado a elementos que hacen del dolor una experiencia individual, modulada por múltiples factores, desde individuales hasta colectivos que permiten la expresión del dolor en el paciente ${ }^{28,29}$.

Evaluar el dolor en los niños con el propósito de reconocerlo y brindar un abordaje clínico es de gran importancia desde cualquier punto de vista, son conocidos los efectos adversos tanto psicológicos como fisiológicos que puede generar el dolor durante la fase aguda en los pacientes ${ }^{11}$. En relación con lo anterior, en el presente estudio el 78,1\% de los pacientes y/o acudientes manifestaron que algún miembro del personal asistencial había indagado sobre dolor en el paciente en las $24 \mathrm{~h}$ previas y el $71,1 \%$ de los pacientes tenían registro sobre la presencia o no de dolor en ese mismo periodo de tiempo, si bien documentar la valoración del dolor no es obligatorio, hace parte del formato de historia clínica de la institución. Hallazgo similar a lo publicado por Zhu y cols. en Canadá en el año 2012 quienes describen que el $63 \%$ de los pacientes tenían al menos una evaluación documentada del dolor en las $24 \mathrm{~h}$ previas ${ }^{30}$.

Para el manejo del dolor en los niños, los equipos de salud deben conocer las herramientas de evaluación 
del dolor y tener la capacidad de elegir un abordaje terapéutico seguro y efectivo ${ }^{31}$. Del total de pacientes, el 53,5\% tenían prescripción analgésica, de los cuales en el $94 \%$ consistía en prescripción farmacológica y en el $6 \%$ farmacológica y no farmacológica; evidenciando el bajo uso de estrategias terapéuticas no farmacológicas (medios físicos y técnicas de inmovilización) o terapias complementarias para el manejo del dolor en pacientes de cualquier grupo de edad, las cuales han demostrado ser eficaces para disminuir el dolor, la angustia y la ansiedad que se puede generar en el ámbito intrahospitalario a través de un enfoque integral en el alivio del dolor adaptadas a los pacientes según la edad y etapa de desarrollo ${ }^{25,32}$.

Así mismo, se documentó que el 23,5\% de los pacientes y/o cuidadores desconocían si tenían alguna prescripción analgésica, lo cual resalta la función que tiene el personal asistencial en explicar el abordaje terapéutico y el derecho que tiene cada paciente de estar informado al respecto. Al mismo tiempo, se evidenció similitud entre el porcentaje de pacientes que creían estar recibiendo algún tipo de analgesia y pacientes que realmente tenían prescripción analgésica, que fue del $54 \%$ y $53,5 \%$, respectivamente.

De igual forma, los pacientes que tenían prescripción analgésica, el 61\% recibían monoterapia y solo el $3 \%$ tenían tres medicamentos analgésicos indicados; siendo el acetaminofén el medicamento más utilizado en el $78 \%$ de pacientes, seguido del metamizol en el $48 \%$ y representando el $33,8 \%$ del total de prescripciones. Se evidencia el amplio uso del metamizol, medicamento que ha sido restringido en muchos países como por ejemplo Estados Unidos y parte de los países miembros de la Unión Europea, basado en efectos adversos graves atribuidos al medicamento, como la agranulocitosis, lo cual genera controversia frente a su uso. En relación con esta situación, una revisión sistemática de la literatura de Andrade y cols. sobre la seguridad del metamizol y sus posibles efectos adversos, especialmente la agranulocitosis, el sangrado intestinal y la hepatotoxicidad, se encontró que la mayoría de los estudios que evaluaron la agranulocitosis demostraron un mayor riesgo asociado con metamizol, con diferencias en las estimaciones de riesgo relativo (RR) que varía de 1,5 (95\% IC, 0,8-2,7) a 40,2 (95\% IC, 14,7$113,3)$ y respaldan la necesidad de más investigaciones para cuantificar mejor los riesgos potenciales asociados al medicamento en relación a la dosis, duración de la terapia, vía de administración o terapia previas y si existían riesgos ${ }^{33}$.

En estudios previos muestran una amplia variación geográfica en la incidencia de agranulocitosis inducida por metamizol ${ }^{34}$ y esto podría explicarse en parte por las diferencias en los patrones de uso de metamizol en términos de dosis, duración y medicamentos conco- mitantes ${ }^{35}$. Un estudio Latinoamericano ${ }^{36}$ documentó una incidencia global de agranulocitosis de 0,38 casos por 1 millón de habitantes-año y concluye que la agranulocitosis inducida por fármacos es muy baja para considerarle un problema de salud pública.

Así mismo, Moreno D. y cols. concluyen que la incidencia de agranulocitosis es variable pero probablemente infrecuente y describen una mortalidad global de 0,5 por millón de habitantes al año ${ }^{37}$. En definitiva, en Colombia el metamizol es ampliamente utilizado para el alivio del dolor ${ }^{38}$ y cuenta con registro vigente otorgado por el Instituto Nacional de Vigilancia de Medicamentos y Alimentos (INVIMA) para su uso.

En este sentido, las intervenciones médicas deben ser seguras y guiadas por condiciones propias del paciente y su entorno, con el objetivo de aliviar el dolor, donde la analgesia debe ser usada con un enfoque gradual o en escalera según la intensidad del dolor, iniciando con analgésicos no opioides y escalando a opioides y analgésicos adyuvantes con efecto analgésico creciente ${ }^{32}$. Con respecto a éste tópico, para el abordaje del dolor moderado a severo, los fármacos no opioides se asocian a fármacos opioides brindando analgesia multimodal para mejorar la eficacia analgési$\mathrm{ca}$, minimizar las dosis de los medicamentos y reducir los efectos adversos, siendo los opioides los fármacos de elección para el tratamiento del dolor moderado a severo tanto en adultos como en niños ${ }^{39,40}$.

Los opioides intravenosos se reservan para el alivio del dolor intenso a través de titulación de dosis; la morfina y el fentanilo son los que se usan con más frecuencia en la población pediátrica ${ }^{32}$. En el estudio, se documenta dolor de intensidad severa en el 9,7\% de los pacientes álgicos al momento de la evaluación y en el 12,5\% de pacientes que habían experimentado dolor en las $24 \mathrm{~h}$ previas a la evaluación, asociado a un bajo uso de medicamentos opioides, solo en el $2 \%$ de los que tenían prescripción farmacológica.

Por último, la Organización Mundial de la Salud (OMS) recomienda la vía oral como la mejor forma para la administración de medicamentos a los niños en la medida de lo posible ${ }^{18}$, está vía de administración se utilizó en el $81 \%$ de los pacientes que tenían prescripción analgésica, pero es llamativo además que la vía parenteral periférica se utilizó en el 52\% de ellos, situación que hace necesario analizar en detalle los procesos de prescripción en la institución; además el 9,15\% de las prescripciones de analgesia farmacológica fueron para administración sin horario y/o a demanda del paciente, contrario a lo propuesto por la OMS. Finalmente, se recomienda la implementación de estrategias y protocolos relacionados con la prevención, evaluación y tratamiento del dolor en instituciones de salud a favor de un abordaje integral del paciente pediátrico.

En conclusión, es imprescindible la identificación 
y el manejo adecuado del dolor en el paciente pediátrico, dado que su prevalencia en población pediátrica hospitalizada en general es alta y más frecuente en pacientes ingresados por cirugía y/o trauma, además se reporta baja prescripción de analgésicos opioides en el entorno pediátrico para el manejo habitual del dolor de intensidad moderado y severo, indicándose preferentemente como analgesia de rescate. Así mismo, un empleo frecuente de metamizol como analgésico a pesar de su controversial uso en diferentes países del mundo, por lo cual se hace necesario más estudios al respecto.

\section{Responsabilidades Éticas}

Protección de personas y animales: Los autores declaran que los procedimientos seguidos se conformaron a las normas éticas del comité de experimentación humana responsable y de acuerdo con la Asociación Médica Mundial y la Declaración de Helsinki.

Confidencialidad de los datos: Los autores declaran que han seguido los protocolos de su centro de trabajo sobre la publicación de datos de pacientes.

\section{Derecho a la privacidad y consentimiento informa-} do: Los autores han obtenido el consentimiento informado de los pacientes y/o sujetos referidos en el artículo. Este documento obra en poder del autor de correspondencia.

\section{Conflicto de intereses}

El autor declara no tener conflicto de intereses.

\section{Referencias}

1. Hudspith MJ. Anatomy, physiology and pharmacology of pain. Anaesth Intensive Care Med. 2019;20(8):419-25.

2. Whitehead K, Papadelis C, LaudianoDray MP, et al. The Emergence of Hierarchical Somatosensory Processing in Late Prematurity. Cereb Cortex. 2019;29(5):2245-60.

3. Bembich S, Cont G, Causin E, et al. Infant analgesia with a combination of breast milk, glucose, or maternal holding. Pediatrics. 2018;142(3):e20173416.

4. AAP committee on fetus and newborn and section on anesthesiology and pain medicine. Prevention and Management of Procedural Pain in the Neonate: An Update. Pediatrics. 2016;137(2):e20154271.

5. Perry M, Tan Z, Chen J, et al. Neonatal Pain: Perceptions and Current Practice. Crit Care Nurs Clin North Am. 2018;30(4):549-61.

6. Carr DB. Pain Is a Public Health Problem - What Does That Mean and Why Should We Care? Pain Med. 2016;17(4):626-7.

7. Lundeberg S. Pain in children - are we accomplishing the optimal pain treatment? Pediatr Anesth. 2015;25(1):8392.

8. Cousins MJ, Lynch ME. The Declaration Montreal: Access to pain management is a fundamental human right. Pain. 2011;152(12):2673-4.

9. Karcioglu O, Topacoglu H, Dikme O, et al. A systematic review of the pain scales in adults: Which to use? Am J Emerg Med. 2018;36(4):707-14.

10. Short S, Pace G, Birnbaum C. Nonpharmacologic Techniques to Assist in Pediatric Pain Management. Clin Pediatr Emerg Med. 2017;18(4):256-60.

11. Brand $\mathrm{K}, \mathrm{Al}$-Rais A. Pain assessment in children. Anaesth Intensive Care Med. 2019;20(6):314-7.

12. Noel M, Chambers CT, McGrath PJ, et al. The influence of children's pain memories on subsequent pain experience. Pain. 2012;153(8):1563-72.

13. Failo A, Giannotti M, Venuti P. Associations between attachment and pain: From infant to adolescent. SAGE Open Med. 2019;7:205031211987777.

14. Leyva Carmona M, Torres Luna R, Ortiz San Román L, et al. Documento de posicionamiento del Grupo Español para el Estudio del Dolor Pediátrico (GEEDP) de la Asociación Española de Pediatría sobre el registro del dolor como quinta constante. An Pediatr. 2019;91(1):58.e158.e7.

15. Annequin D, Ngo J. Evaluación y tratamiento del dolor en recién nacidos, niños y adolescentes. EMC - Tratado Med. 2019;23(1):1.6.

16. Moraes M, Zunino C, Duarte V, et al. Evaluación de dolor en niños hospitalizados en servicios de salud públicos y privados de Uruguay. Arch Pediatr Urug. 2016;87(3):198-209.

17. Rojas M, Vanoli N, Mimbacas I, et al. Dolor en niños y adolescentes hospitalizados en un centro de referencia. Rev Chil Pediatría 2018;89(1):67-73.

18. Organización Mundial de la Salud. Directrices de la OMS sobre el tratamiento farmacológico del dolor persistente en niños con enfermedades médicas. Ginebra 2012.

19. Wong C, Lau E, Palozzi L, et al. Pain management in children: Part 1 - Pain assessment tools and a brief review of nonpharmacological and pharmacological treatment options. Can Pharm J (Ott). 2012;145(5):222-5.

20. Bieri D, Reeve RA, Champion GD, et al. The faces pain scale for the self-assessment of the severity of pain experienced by children: Development, initial validation, and preliminary investigation for ratio scale properties. Pain. 1990;41(2):139-50.

21. Hicks CL, von Baeyer CL, Spafford PA, et al. The Faces Pain Scale-Revised: toward a common metric in pediatric pain measurement. Pain. 2001;93(2):173-83.

22. Birnie KA, Hundert AS, Lalloo C, et al. Recommendations for selection of selfreport pain intensity measures in children and adolescents. Pain. 2019;160(1):5-18.

23. López-Guzmán J, Pazos-Alvarado RE, Moyao-García D G-NA. Prevalencia e incidencia del dolor en los pacientes hospitalizados en el Hospital Infantil de México «Federico Gómez» en un período de seis meses. Rev Mex Anestesiol. 2013;36(2):93-7.

24. Krauss BS, Calligaris L, Green SM, et al. Current concepts in management of pain in children in the emergency department. Lancet. 2016;387(10013):83-92.

25. Young VB. Effective Management of Pain and Anxiety for the Pediatric Patient in the Emergency Department. Crit Care Nurs Clin North Am. 2017;29(2):205-16.

26. Liossi C, Howard RF. Pediatric Chronic Pain: Biopsychosocial Assessment and Formulation. Pediatrics. 2016;138(5):e20160331.

27. Friedrichsdorf SJ, Postier A, Eull D, et al. Pain Outcomes in a US Children's Hospital: A Prospective Cross-Sectional Survey. Hosp Pediatr. 2015;5(1):18-26. 
28. McCormick T, Frampton C. Assessment of acute and chronic pain. Anaesth Intensive Care Med. 2019;20(8):405-9.

29. Young KD. Assessment of Acute Pain in Children. Clin Pediatr Emerg Med. 2017;18(4):235-41.

30. Zhu LM, Stinson J, Palozzi L, et al. Improvements in pain outcomes in a Canadian pediatric teaching hospital following implementation of a multifaceted knowledge translation initiative. Pain Res Manag. 2012;17(3):173-9.

31. Manworren RC, Stinson J. Pediatric Pain Measurement, Assessment, and Evaluation. Semin Pediatr Neurol. 2016;23(3):189-200.

32. Gaglani A, Gross T. Pediatric Pain
Management. Emerg Med Clin North Am. 2018;36(2):323-34.

33. Andrade S, Bartels DB, Lange R, et al. Safety of metamizole: a systematic review of the literature. J Clin Pharm Ther. 2016;41(5):459-77.

34. Shah RR. Metamizole (dipyrone)-induced agranulocytosis: Does the risk vary according to ethnicity? J Clin Pharm Ther. 2019;44(1):129-33.

35. Ibáñez L, Vidal X, Ballarín E, et al. Agranulocytosis associated with dipyrone (metamizol). Eur J Clin Pharmacol. 2005;60(11):821-9.

36. Hamerschlak N, Maluf E, Biasi Cavalcanti A, et al. Incidence and risk factors for agranulocytosis in Latin American countries - The Latin Study: A multicenter study. Eur J Clin Pharmacol.
2008;64(9):921-9.

37. Moreno DA, Echeverry MA. Current controversies on the clinical use of dipyrone: Safe alternative? Colomb J Anesthesiol. 2019;47(4):261-3.

38. Fuentes-Losada LM, Vergara-Amador E, Laverde-Cortina R. Pain management assessment in children with limb fractures in an emergency service. Rev Colomb Anestesiol. 2016;44(4):305-10.

39. Cunningham ME, Vogel AM. Analgesia, sedation, and delirium in pediatric surgical critical care. Semin Pediatr Surg. 2019;28(1):33-42.

40. Martin L, Jimenez N, Lynn AM. Farmacología del desarrollo de analgésicos opioides y no esteroideos en neonatos e infantes. Rev Colomb Anestesiol. 2017;45(1):72-9. 